\title{
- Research Paper Effect and Mechanism of Jiawei Shaomu Decoction on a Rat Model of Post-Stroke Spasm
}

\author{
XIAOMENG WANG, QUANE WU, S. HOU1 AND Y. HAN* \\ School of Basic Medical Sciences, ${ }^{1}$ Graduate School, Hebei University of Chinese Medicine, Shijiazhuang, Hebei 050091, \\ China
}

Wang et al.: Effect of Jiawei Shaomu Decoction on Post-Stroke Spasm

\begin{abstract}
To establish a blood stasis model of rats and its subsequent stroke spasm model and to investigate the therapeutic effects of Jiawei Shaomu decoction on muscle spasm in blood stasis type stroke rats using experimental technical means including pathology, immunity and molecular biology. A total of 100 rats were divided into blank group (rats without modeling), blood stasis group (rats with blood stasis syndrome), stroke group (rats with blood stasis type stroke), positive drug group (rats with baclofen+blood stasis type stroke), Shaomu group (rats with Shaoyao Mugua decoction+blood stasis type stroke) and Jiawei group (rats with Jiawei Shaoyao Mugua decoction+blood stasis type stroke). Then, after treatment by gavage, the weightbearing swimming time, grip strength and coagulation indexes were detected to evaluate the therapeutic effects of Jiawei Shaomu decoction on blood stasis type stroke rats. The therapeutic effects of Jiawei Shaomu decoction on muscle spasms after stroke were analyzed by examining the electromyography, antioxidant indexes, biochemical indexes and inflammatory factors in the gastrocnemius muscle of rats in each group. The protein content and gene expression related to acetylcholine receptor signal pathway were detected to analyze the mechanism by which Jiawei Shaomu decoction inhibits rats with post stroke spasm. Shaomu decoction and Jiawei Shaomu decoction can effectively resist the inflammatory reaction that appears in motor tissues after stroke by increasing antioxidant capacity. Shaomu decoction and Jiawei Shaomu decoction achieve their effects in treating muscle spasms after stroke by inhibiting the extracellular signal regulated kinase signal pathway and downregulating acetylcholine receptor concentrations on the muscle cell membrane. Jiawei Shaomu decoction is superior to Shaomu decoction in treating post stroke spasm of blood stasis type.
\end{abstract}

Key words: Blood stasis syndrome, Jiawei Shaomu decoction, stroke, spasm, acetylcholine receptor

Spastic paraparesis is a frequent sequela of stroke. Traditional Chinese medicine considers that the spastic paralysis state exhibited by patients after stroke is mainly characterized by unfavorable flexion extension of fascicles and joints and often involuntary contraction of muscles. Due to the irregular diet and life habits of modern people, blood stasis is a pathological factor throughout the whole process before and after stroke onset. Clinical studies have shown that paeony and papaya decoction can be used to improve the symptoms of gastrocnemius muscle spasms, but there have been no reports on the effects of paeony and papaya decoction on limb spasms after stroke. In this paper, Ligusticum wallichii and Achyranthes bidentata with analgesic and blood activating effects were added to paeony and papaya decoction, which was configured to taste Jiawei paeony and papaya decoction to make gavage in a rat model of stroke spasm and the effects on muscle tone and neurotransmitters in a rat model of spasm were investigated, with a view to providing a scientific reference for the application of paeony and papaya decoction in the rehabilitation of patients with stroke spasm ${ }^{[1]}$.

\section{MATERIALS AND METHODS}

Experimental materials:

Animal experiments: 90 male healthy Sprague Dawley (SD) rats of Specific Pathogen Free (SPF) grade were selected, weighing 190-210 g, provided by Heilongjiang University of Chinese Medicine with license number: SCXK (H) 2018-007. The rearing environment temperature was $25 \pm 2^{\circ}$ and relative humidity $60 \pm 5$ $\% .7 \mathrm{~d}$ before the start of the experiment, the rats were adaptively raised in the feeding house, with light hours between 8:00 and 20:00 a d and free access to water and diet.

*Address for correspondence

E-mail: lilygod_m@163.com 
Medicinal materials: For experimental use medicinal materials drinker tablets (fig. 1) $12 \mathrm{~g}$ of Paeonia lactiflora Pallas, $6 \mathrm{~g}$ of Chaenomeles sinensis, $6 \mathrm{~g}$ of Ligusticum chuanxiong Hort and $6 \mathrm{~g}$ of Achyranthes bidentata were purchased from the First Affiliated Hospital of Heilongjiang University of Chinese Medicine and oven dried at $50^{\circ}$. After the Paeonia lactiflora Pallas and Chaenomeles sinensis were configured into a prescription in a 2:1 ratio, they were decocted by adding water at a solid-liquid ratio of 1:10 for two times, each decocting for $90 \mathrm{~min}$ and the filtered juice was taken and mixed and concentrated, so that $1 \mathrm{ml}$ of the medicine solution was equivalent to 1 $\mathrm{g}$ of the crude medicine to make the experimental use of Shaomu (Paeonia lactiflora Pallas+Chaenomeles sinensis) medicine liquid ${ }^{[2]}$. At the same time, drinker tablets of Paeonia lactiflora Pallas, Chaenomeles sinensis, Achyranthes bidentata and Ligusticum chuanxiong Hort were combined in a 2:1:1:1 ratio to make an additive prescription and after being cooked as described above, they were concentrated so that 1 $\mathrm{ml}$ of the filtrate corresponded to $1 \mathrm{~g}$ of crude drugs to make an experimental solution of the Jiawei Shaomu medicine liquid ${ }^{[3]}$.

Experimental reagents: Chloral hydrate was purchased from Merck \& Co., USA, prothrombin time kit, activated partial thromboplastin time kit, plasma thrombin time kit and fibrinogen kit were purchased from Beijing Strong Biotechnologies, Inc.; n-heptane, glacial acetic acid, hydrochloric acid, o-phthalaldehyde, sodium periodate, sodium tetraborate and sodium sulphite were purchased from Tianjin Chemical Reagent Co., Ltd; Coomassie brilliant blue was purchased from Nanjing Jiancheng Co., Ltd.; Creatine Kinase (CK) Enzyme-Linked Immunosorbent Assay (ELISA) assay kit, Lactate Dehydrogenase (LDH) ELISA assay kit, Malondialdehyde (MDA) ELISA assay kit, Superoxide Dismutase (SOD) ELISA assay kit and Tumor Necrosis Factor Alpha (TNF- $\alpha$ ) ELISA kit was purchased from R\&D (USA); Ribonucleic Acid (RNA) extraction kit, fluorometric quantitative Polymerase Chain Reaction (PCR) kit, reverse transcription kit, Polyvinylidene Fluoride (PVDF) membrane was purchased from Dalian Takara (China); PVDF membrane was purchased from US Summus (USA); acrylamide, methylene diacrylamide, ammonium persulfate, tetramethylethylenediamine, glycine and tris(hydroxymethyl)aminomethane were purchased from Sigma (USA); PageRuler ${ }^{\mathrm{TM}}$ Prestained Protein Ladder was purchased from Thermo(USA); Sodium Dodecyl Sulfate (SDS) and tween20 were purchased from biotoPPed (USA), westernbloting and Immunoprecipitation lysate, Phenyl Methyl Sulfonyl Fluoride (PMSF), primary antibody dilutions, primary and secondary antibody removal solutions and secondary antibodies were purchased from Beyotime Inc. (Beijing, China).

Experimental instruments: Small animal noninvasive pulse oximetry (Starr, USA); physiological recorder (BIOPAC, USA); cryogenic centrifuge (Sigma, USA); hemagglutination apparatus (Perlong Medical Co., Beijing, China); fully automated blood rheometer (Perlong Medical Co., Beijing, China); microplate reader (TECAN, USA); electromyograph (Stoelting, USA); microplate reader (TECAN, USA); transmission electron microscopy (Electron Co., Ltd., Japan); cryogenic centrifuge (Sigma, USA); electronic balance (Seldoms, Switzerland); $-80^{\circ}$ cryogenic refrigerator (Sanyo ice maker Japan; Glant UAS ultrapure water meter (Millipore, USA); gel imaging system (UVP, USA); cryogenic centrifuge (Sigma, Germany); highspeed centrifuge (Shanghai Anting); general PCR instrument (mjP100, USA); horizontal electrophoresis system (Beijing Liuyi, China); precision balance (Sartorius, Switzerland); acidometer (Odron, USA); UV spectrophotometer (Shimadzu UV-2401, Japan); fluorescence quantitative PCR (ABI7500, USA) ${ }^{[4]}$.

\section{Experimental methods:}

Establishment of model of post-stroke spasm: A total of 78 rats were randomly selected for the experiment and blood stasis syndrome rat modeling was performed by using a high-fat diet referring to the "Pharmacology Research Methodology of Traditional Chinese Medicine" and modeling of rats with blood stasis syndrome by Wang et al. A blood stasis syndrome rat model was developed by feeding a high-fat diet (1\% cholesterol, $7.5 \%$ lard, $0.2 \%$ propylthiouracil, $0.3 \%$ bile salts, $10 \%$ egg yolk powder and $81 \%$ basal diet) for $28 \mathrm{~d}$. A total of 60 blood stasis syndrome rats were obtained and the Middle Cerebral Artery Occlusion (MCAO) model was performed to model blood stasis stroke rats. The rats were first anesthetized and pending successful anesthesia, the rats were secured in a supine position with a neck prepped incised at the median softest point of the rat's neck, stripped of muscle groups from the neck toward the interior and the common, internal and external carotid arteries on the right side of the rat's neck were located and separated by forceps ${ }^{[5,6]}$. The common artery clip and the proximal end of the external carotid artery were then ligated with a No. 1 silk suture, while the distal end of the external 
carotid artery was temporarily closed with a vascular clip, then a small orifice was opened at the distal end of the external carotid artery and a pre-prepared wire plug was inserted, the ligature at the proximal end was tied and then the vessel clip was removed and the wire plug was slowly advanced through the external carotid artery bifurcation into the internal carotid artery, followed by adjustment of the wire plug to the bottom right, the bifurcation of the external carotid artery was inserted back, the thread of the plug was fixed and after the vessel at the proximal end of the middle cerebral artery was closed, the rats' wound was sutured layer by layer, the wound was disinfected, kept the rats in a normal cage after the rats recovered from the operation, the rats were scored for the condition of neurological impairment $24 \mathrm{~h}$ after the operation and the rats with 0 points that the modeling was unsuccessful were eliminated. Experiments MCAO modeling rats were scored referring to the method of Zea Longa. Eight rats died during the MCAO modeling operation and during recovery, 5 rats scored 0 for unsuccessful modeling, a total of 47 rats with blood stasis type stroke were obtained and 40 rats with similar body conditions were selected for subsequent experiments.

Design and grouping experiment: The experiment used a univariate design and the rats were divided into blank group, blood stasis group, stroke group, positive drug group, Shaomu group and Jiawei Shaomu group depending on the experimental treatment. Each group consisted of 10 rats and the $28^{\text {th }} \mathrm{d}$ of stroke modeling was the $1^{\text {st }} \mathrm{d}$ of the experiment and the experiment lasted for $28 \mathrm{~d}$, which were housed the same way as the pre-feeding period during the experimental period. The specific treatments were as follows: In blank group, rats selected without any treatment were made gavage with normal saline at $1.0 \mathrm{ml} / 100 \mathrm{~g}$ daily. The blood stasis group selected the post-stroke spasm model rats and received normal saline solution at $1.0 \mathrm{ml} / 100 \mathrm{~g}$ daily. For the stroke group, the blood stasis type post-stroke spasm model rats were selected and the model rats were given normal saline by gavage every day at $1.0 \mathrm{ml} / 100$ $\mathrm{g}$. The positive drug group received baclofen by gavage daily at $1.35 \mathrm{mg} / \mathrm{kg}$ to the blood stasis stroke model rats. Shaomu group received Shaomu liquid daily by gavage at $1.0 \mathrm{ml} / 100 \mathrm{~g}$ to blood stasis type stroke model rats. Jiawei Shaomu group was intragastrically gavaged with Jiawei Shaomu liquid daily at 1.0 $\mathrm{ml} / 100 \mathrm{~g}$ to blood stasis type stroke model rats. Using a univariate design, rats were divided into four groups according to the experimental treatment: control group, sham-operated group, model group, low-dose group, middle-dose group and high-dose group ( $\mathrm{n}=10$ each), from experimental $\mathrm{d} 1$. The total experimental period $28 \mathrm{~d}$ and maintained the same feeding way as the prefeeding period during the experimental period, with the following specific treatments: Rats without any treatment were chosen for the control group and gavage with normal saline each day according to the ratio of $1.0 \mathrm{ml} / 100 \mathrm{~g}$; in the sham-operated group, normal rats underwent drilling surgery without damage to the internal capsule and normal saline was administered daily at $1.0 \mathrm{ml} / 100 \mathrm{~g}$; the model group received normal saline daily by gavage to the model rats at $1.0 \mathrm{ml} / 100$ $\mathrm{g}$; the low-dose group received the gavage solution of model rats at $0.5 \mathrm{ml} / 100 \mathrm{~g}$ daily; the middle-dose group received the gavage solution of model rats at 1.0 $\mathrm{ml} / 100 \mathrm{~g}$ daily; and the high-dose group received the gavage solution of model rats at $1.5 \mathrm{ml} / 100 \mathrm{~g}$ daily.

\section{Detection indexes and methods:}

Extension amplitude assay: The amplitude of spastic limb extension was measured in rats on experimental d 25 using a physiologic recorder. One end of the electro stimulation needle was inserted into the gastrocnemius muscle of the lower extremity on the spastic side of the rats that was fixed to a scale plate and the other end was inserted into the tail of the rats and a silk thread was tied under the lower extremity on the spastic side. The wire was connected to a physiological recorder and the amplitude $(\mathrm{cm})$ of lower extremity extension in the experimental rats was recorded by timed electrical stimulation of the gastrocnemius muscle at $2 \mathrm{~mA}$.

Weight bearing swimming time assay: On experimental d 25, $10 \mathrm{~g}$ weight-bearing weights were fixed to the rat tail tip, placed in distilled water about $50 \mathrm{~cm}$ height in a swimming tank at $150 \mathrm{~cm}$ long, $80 \mathrm{~cm}$ wide and $80 \mathrm{~cm}$ high, water temperature $22^{\circ}$ $25^{\circ}$, rats with tail tip anchored weights were placed in a swimming tank, the rats were allowed to swim to exhaustion, taking $10 \mathrm{~s}$ above when the rat's head cannot emerge from the water as the standard and after recording the swimming time, the rats were scooped out, blotted dry and placed back to feeding cages and give them normal feeding.

Grip strength assay: On experimental d 25, when the rats were in calm condition, the rats were grasped to be placed on the grip strength board and after the rats reached to grasp the board vigorously, the rat tail was quickly pulled from the direction of parallel rats, the grasping value was read, repeated 3 times and the average value was taken. 
Pulse rate assay: On the $25^{\text {th }} \mathrm{d}$ of the experiment, the rats were anesthetized and the rat's pulse was measured at its plateau using a small animal noninvasive pulse oximeter and an effective value of 10 was recorded for each rat and averaged.

Hemorheological assay: On the $25^{\text {th }} d$ of the experiment, the rats were anesthetized, blood was withdrawn from the rat abdominal artery using heparin tubes and the hemorheological indexes were detected using a fully automated blood rheometer.

Coagulation index testing: On the $25^{\text {th }} \mathrm{d}$ of the experiment, the rats were anesthetized, blood was collected from the rat abdominal artery using an anticoagulant tube, centrifuged at $1500 \mathrm{rpm}$ at low temperature and the supernatant was collected to measure the prothrombin time, activated partial prothrombin time, plasma thrombin time and fibrinogen in the rat serum according to the operation of the kit instructions.

Rat gastrocnemius muscle electromyography assay: At the end of modeling in both models, 10 rats from each group were fixed to the electromyographic recorder, needle electrodes were inserted at the fullest point of the right gastrocnemius muscle of the rats, the sensors were fixed and the rat gastrocnemius electromyographic values were recorded and each rat was detected three times and averaged.

Gastrocnemius antioxidant index assay: At the end of modeling in both models, 2 rats from each group were anesthetized, the right gastrocnemius muscle was removed, $1.0 \mathrm{~g}$ of gastrocnemius muscle sample was put into an Eppendorf (EP) tube of $20 \mathrm{ml}, 10 \mathrm{ml}$ of normal saline was added, the EP tube was placed into ice, homogenized with a homogenizer and centrifuged $3000 \mathrm{rpm}$ at low temperature for $15 \mathrm{~min}$ to extract the supernatant. Total protein in tissue homogenates was detected by Coomassie brilliant blue assay and SOD and MDA contents were detected by ELISA kits.

Gastrocnemius muscle biochemical index measurement: After the end of modeling in both models, use the supernatant prepared. Total protein was detected by Coomassie brilliant blue assay in tissue homogenates and Interleukin-1 Beta (IL-1 $)$ and TNF- $\alpha$ were detected in tissue homogenates using ELISA kits.

Inflammatory factor assay in gastrocnemius muscle: After the end of modeling in both models, use the supernatant prepared. Total protein in tissue homogenates was detected by Coomassie brilliant blue assay and CK and LDH levels in tissue homogenates were detected by ELISA kits.

Gastrocnemius muscle morphological observation: At the end of modeling in both models, 10 rats from each group were anesthetized, the left gastrocnemius muscles were removed and the samples were cut into 1 $\mathrm{mm}$ cubes, after fixed with glutaraldehyde, dehydrated, embedded, cut into ultrathin sections and examined by transmission electron microscopy for pathological changes in the morphology of myofibrils, mitochondria and sarcoplasmic reticulum in the gastrocnemius muscle tissues.

\section{Detection of Acetylcholine Receptor (AChR) signal pathway related gene expression:}

At $28 \mathrm{~d}$ of the experiment, after rats were killed by spinal amputation, the left gastrocnemius muscle samples of the rats were quickly removed and placed into pre-treated EP tubes, quickly frozen with liquid nitrogen and stored in a $-80^{\circ}$ freezer. Tissue samples were collected for extraction of total RNA.

Internal reference gene primers were designed according to the gene sequence on the National Center for Biotechnology Information Genebank, the amplified fragment length was 100-300 bp and the specific information of the primers was as follows: Glyceraldehyde-3-phosphate dehydrogenase (GAPDH) (reference genes) 5'TTCAACGGCACAGTCAAG3'; AchR 5'TACTCAGCACCAGCATCA3', 5 ' G G C T G T G A A C T C T G T C T C 3', $5^{\prime} \mathrm{G} \mathrm{G} \mathrm{C} \mathrm{A} \mathrm{T} \mathrm{C} \mathrm{T} \mathrm{C} \mathrm{C} \mathrm{T} \mathrm{T} \mathrm{C} \mathrm{C} \mathrm{A} \mathrm{T} \mathrm{T} \mathrm{C} \mathrm{C} \mathrm{3';}$ Extracellular signal-regulated kinase $1 / 2$ (ERK) 5'CTTCAATGCGACCTTCCA3', 5' T A C A A C G G C A A C T C C T T A G 3'; Ras 5'CTCAACCGCTTGCTGTAT3', 5 ' G T A A T C A T C C G C C A C T C A T 3'; Raf 5'TCAAGCAAGAGAAGAAGGA3', 5 ' C C G T A A G T C A T C G C C A T T 3' ; Mek 5'CTCTTCTTCCACCTGTC3', $5^{\prime}$ G C C A T A G T C G T T G T C C T C 3' ; c-fos 5'TTTGGCTCCTGGATTAC3', 5'CAGCGAACTTCCCTTAC3'.

Total RNA extraction, Complementary DNA (cDNA) reverse transcription and quantitative fluorescent PCR were performed according to the kit instructions. The experiment used a double standard curve to obtain the true concentrations of the reference and target genes in the samples, which were first through the standard curve and then corrected by the internal reference genes. From the amplification curve, it can be seen that the instrument can effectively detect the amplification 
of the gene to be tested; the dissolution curve has only one single peak, indicating that the primer specificity is better and can be used to perform quantitative experiments; the linear regression rate of the standard curve is all above $99.0 \%$, indicating that in this concentration range of the sample gene to be tested, it is linear and statistical analysis can be performed.

\section{Detection of $\mathrm{AChR}$ pathway related proteins:}

Protein extraction: Thaw the Western and Immunoprecipitation (IP) cell lysates taken from the freezer at $-20^{\circ}$ and mix. Take $1000 \mu 1$ to add PMSF 10 $\mu 1$ to a $1.5 \mathrm{ml}$ EP tube. Weigh $100 \mathrm{mg}$ of the sample in a lysis solution containing PMSF. In this procedure the EP tube is always embedded in crushed ice. The homogenizer tries to crush the tissue and place it on ice to lyse. After lysis for $30 \mathrm{~min}$, the middle of the three layers (supernatant), i.e., the extracted protein was carefully removed to a new $1.5 \mathrm{ml}$ EP tube by centrifugation at $12000 \mathrm{rpm}$ for $10 \mathrm{~min}$. The protein concentration was directly detected by the detector. Aliquot samples, store at $-80^{\circ}$.

\section{Sodium Dodecyl Sulphate-Polyacrylamide Gel} Electrophoresis (SDS-PAGE) electrophoresis: Complete separation gel according to the SDS-PAGE gel kit instructions operation to prepare $12 \%$ gel, at 10-15 min, a crease will appear between the water gels, at which time the gel has solidified, pour off $1 / 3$ of the ultrapure water, residual liquid was carefully blotted with filter paper, $5 \%$ concentrate was formulated, after completion, insert toothcombs and put horizontally for about $15 \mathrm{~min}$ waiting for gelation. The volume of the corresponding protein solution was calculated as the loading capacity based on the concentration of the protein measured. Pipette to a $0.5 \mathrm{ml}$ EP tube, add $1 \times$ SDS loading buffer, briefly centrifuge and place into the PCR machine for protein denaturation at $100^{\circ}$ for $10 \mathrm{~min}$. After the concentrated gel had solidified, remove the gel plate, flush out the residue, place the electrophoresis tank, add electrophoresis solution, inner tank was poured full or at least a short glass plate with the inside submerged at the liquid level, the outer tank was poured to the tick mark, gently pull the comb in the electrophoresis solution, inject the prepared protein sample into the lanes with a pipette, load $10 \mu 1$ per well and finally add prestained protein marker, add $1 \times$ SDS loading buffer liquid to extra blank lanes. Switch on the electrophoresis apparatus. Electrophoresis was started by running the spacer gel, which could be set to a voltage of $60 \mathrm{~V}$ and after bromophenol blue entered the separating gum, the voltage was changed to $100 \mathrm{~V}$ until the running out of the separation gel was terminated and the process was 1.5-2.0 $\mathrm{h}$.

Transfer membrane: End the electrophoresis, remove the glass plates, flush off the surface residual gel, pry the glass plates gently through a plastic sheet, remove the short plates, excise the concentrated gel, cut the separation gel according to the size of the molecular weight of the protein of interest and mark the large marker at the upper right corner to facilitate distinguishing the positive and negative sides of the membrane and equilibrate in the transmembrane solution for $10 \mathrm{~min}$. Prepare to tile the "sandwich" with the equilibrated thick filter paper, PVDF membrane, separation gel, thick filter paper placed sequentially from bottom up and after everything was in place, covered with the cover switching on the power supply for transferring membrane. Generally, $15 \mathrm{~V}, 15-20 \mathrm{~min}$.

Block: At the end of the transferring membrane, the PVDF membrane was moved into a flat dish containing Tris Buffered Saline with Tween (TBST) buffer and washed twice with TBST for $10 \mathrm{~min}$ each. After washing and transferring the membrane into a flat dish full of blocking solution, the PVDF membrane should be completely submerged below the liquid level, placed on a horizontal shaker and closed for $2 \mathrm{~h}$ with slow shaking.

Hybridization: After blocking, the PVDF membrane was washed twice with TBST for $10 \mathrm{~min}$ each. Afterwards place the membrane carefully in the hybridization bag, close by a sealer and leave one side open with the formulated primary antibody, sealed. Place in $4^{\circ}$ freezer overnight. Remove out the next morning, move to a flat dish containing TBST solution and wash four times for $10 \mathrm{~min}$ each, secondary to washing with Tris Buffered Saline (TBS) for 10 min. Prepare for secondary antibody incubation. The membrane and secondary antibody were enclosed into a hybridization bag, shaken on a horizontal shaker and incubated at room temperature. After $1 \mathrm{~h}$, secondary antibodies were eluted.

Chemiluminescence: Chemiluminescence, developed. Place PVDF membrane protein faces up in a glass plate or cropped parafilm bag and blot the liquid with filter paper. After mixing with equal volumes of two reagents, $\mathrm{A}$ and $\mathrm{B}$ of Electrochemiluminescence (ECL) liquid, make them homogeneous, drop onto PVDF membrane and expose after about 1 min to allow the luminescent liquid to react sufficiently with proteins and save the images. 


\section{Statistical methods:}

Statistical Package for the Social Sciences (SPSS) 23.0 software was used for analysis and Analysis of Variance (ANOVA) was used for data comparative analysis, all results were expressed as mean \pm standard deviation $(\overline{\mathrm{x}} \pm \mathrm{s})$ and $\mathrm{p}<0.05$ was used to represent significant difference.

\section{RESULTS AND DISCUSSION}

The results of the lower limb extension amplitude determination of the rats are shown in Table 1. The extension amplitude of the lower limbs was normal in the blank group, slightly lower in the blood stasis group than in the blank group and significantly lower in the stroke group than in the blank group and blood stasis group ( $\mathrm{p}<0.05)$. The extension amplitude of the lower limbs in the positive drug group was significantly lower than that of the blank group $(\mathrm{p}<0.05)$, but significantly higher than that of the stroke group $(p<0.05)$. The extension amplitude of the lower limbs was significantly higher $(\mathrm{p}<0.05)$ in the Shaomu group than in the stroke group, but significantly lower $(p<0.05)$ in the blank group, blood stasis group and positive drug group and higher $(\mathrm{p}<0.05)$ in the Jiawei Shaomu group than in the stroke group, but significantly lower $(p<0.05)$ than in the blank group and blood stasis group.

TABLE 1: LOWER LIMB EXTENSION AMPLITUDE MEASUREMENT IN RATS MEASUREMENT ( $\bar{x} \pm s)$ $(n=10)$

\begin{tabular}{lc}
\hline Group & Extension amplitude $(\mathrm{cm})$ \\
\hline Blank group & $0.49 \pm 0.05$ \\
Blood stasis group & $0.45 \pm 0.04$ \\
Stroke group & $0.22 \pm 0.03^{\Delta *}$ \\
Positive drug group & $0.40 \pm 0.06^{\Delta \sharp}$ \\
Shaomu group & $0.31 \pm 0.04^{\star \star * \circ}$ \\
Jiawei Shaomu group & $0.38 \pm 0.05^{\star \star *}$ \\
\hline
\end{tabular}

Note: ${ }^{\wedge} \mathrm{p}<0.05$ vs. blank group, ${ }^{*} \mathrm{p}<0.05$ vs. blood stasis group, ${ }^{\#} \mathrm{p}<0.05$ vs. stroke group, ${ }^{\circ} \mathrm{p}<0.05$ vs. positive drug group

The comparison of the time of weight-bearing swimming to exhaustion among the rats is presented in Table 2. The longest time of weight-bearing swimming to exhaustion was significantly higher in the blank group than in the blood stasis group and stroke group $(p<0.01)$, whereas the shortest time of weight-bearing swimming to exhaustion was observed in the stroke group and was significantly lower than that in the blood stasis group $(p<0.05)$. Among the three drug treated groups, the weight-bearing swimming time of the positive drug group was the longest, significantly higher $(p<0.05)$ than that of the stroke group, but significantly lower $(p<0.01)$ than that of the blank group, the weightbearing swimming time of the rats in Shaomu group increased $(p<0.01)$ than that of the stroke group, but remained significantly lower $(\mathrm{p}<0.05)$ than that of the rats in the blank group and the weight-bearing swimming time of the rats in the Jiawei Shaomu group, although significantly lower than that of the blank group $(p<0.01)$ and the blood stasis group $(p<0.05)$, significantly higher than the stroke group $(\mathrm{p}<0.05)$.

TABLE 2: WEIGHT-BEARING SWIMMING OF RATS $(\bar{X} \pm s)(n=10)$

\begin{tabular}{lc}
\hline Group & Time $(\mathrm{s})$ \\
\hline Blank group & $1358.15 \pm 108.75$ \\
Blood stasis group & $847.28 \pm 113.88^{\star}$ \\
Stroke group & $698.84 \pm 94.18^{\triangle \star}$ \\
Positive drug group & $790.29 \pm 87.58^{\triangle \#}$ \\
Shaomu group & $724.68 \pm 91.36^{\triangle \#}$ \\
Jiawei Shaomu group & $767.24 \pm 79.84^{\text {^\# }}$ \\
\hline
\end{tabular}

Note: ${ }^{\wedge} \mathrm{p}<0.01$ vs. blank group, ${ }^{*} \mathrm{p}<0.05$ vs. blood stasis group, ${ }^{\#} \mathrm{p}<0.05$ vs. stroke group

The rat grip strength measurement results are shown in Table 3. As can be found from Table 3, the rat grip strength in the blank group was normal and significantly higher than that in the other groups $(\mathrm{p}<0.05)$, while the stroke grip strength was the weakest and significantly lower than that in the blood stasis group $(\mathrm{p}<0.05)$. The grip strength of the positive drug group was significantly higher than that of the blank group but significantly lower than that of the blood stasis group $(p<0.05)$. The grip strength of the rats was significantly higher in Shaomu group than that in stroke group $(p<0.05)$, but significantly lower than that in positive drug group $(p<0.05)$ and Jiawei Shaomu group was significantly higher than that of stroke group $(p<0.05)$ but significantly lower than blood stasis group $(p<0.05)$.

TABLE 3: RAT GRIP STRENGTH ASSAY $(\bar{x} \pm s)(n=10)$

\begin{tabular}{lc}
\hline Group & Grip strength $(\mathrm{g})$ \\
\hline Blank group & $1579.19 \pm 159.28$ \\
Blood stasis group & $1009.54 \pm 162.19^{\star}$ \\
Stroke group & $608.76 \pm 85.38^{\star \star}$ \\
Positive drug group & $843.81 \pm 78.61^{\Delta \star \#}$ \\
Shaomu group & $740.84 \pm 85.69^{\star \star \circ}$ \\
Jiawei Shaomu group & $806.75 \pm 97.38^{\star \star \# \circ}$ \\
\hline
\end{tabular}

Note: ${ }^{\wedge} \mathrm{p}<0.01$ vs. blank group; ${ }^{*} \mathrm{p}<0.01$ vs. blood stasis group; ${ }^{\#} \mathrm{p}<0.05$ vs. stroke group 
A comparison of the coagulation parameters of the rats is shown in Table 4. As can be found from Table 4, although there was a decreasing trend of the coagulation parameters in the model group, it was not significantly different from the blank group and all the treatment groups were also not significantly different from the blank group and model group ( $\mathrm{p}>0.05$ ).

The changes in the electrical potentials of coagulated gastrocnemius muscles of rats are shown in Table 5. As can be seen from Table 5, the voltage values of gastrocnemius muscles of rats in the blank group were significantly higher than those in the two model groups $(p<0.05)$, while the voltage values of the stroke group were significantly higher/lower than those of positive drug group and Jiawei Shaomu group $(\mathrm{p}<0.05)$, the Shaomu group had a tendency to increase the electrical voltage of gastrocnemius muscles of rats with stroke, but the difference was not significant.

The comparisons of antioxidant indexes among rats are shown in Table 6. The gastrocnemius muscle of rats in the blank group showed the best antioxidant level, significantly higher SOD $(\mathrm{p}<0.01)$ and significantly lower MDA $(p<0.01)$ that other groups, significantly lower SOD $(\mathrm{p}<0.01)$ in the stroke group than in the blood stasis group and significantly lower SOD $(p<0.05)$ in the positive drug group than in the Shaomu group and the Jiawei Shaomu group, the MDA level in the Jiawei Shaomu group was significantly lower than that in the model group and the positive drug group $(\mathrm{p}<0.05)$.

The indicators of inflammatory factors in rats are shown in Table 7. As can be seen from Table 7, IL$1 \beta$ and TNF- $\alpha$ in the gastrocnemius muscle of rats in the blank group were significantly lower than those in the model group $(\mathrm{p}<0.05)$, in the blood stasis group were significantly lower than those in the stroke group $(p<0.05), I L-1 \beta$ in the gastrocnemius muscle of rats in each drug administration group was significantly lower than that in the model group $(p<0.05)$ and TNF- $\alpha$ in the gastrocnemius muscle of the positive drug group was also lower than that in the model group $(\mathrm{p}<0.05)$.

TABLE 4: COMPARISON OF COAGULATION INDEXES IN RATS $(\overline{\mathrm{X} \pm s})(\mathrm{n}=10)$

\begin{tabular}{lccccc}
\hline Group & Sample size $(\mathrm{n})$ & PT $(\mathrm{s})$ & APTT $(\mathrm{n})$ & \multicolumn{2}{c}{ Plasma thrombin } \\
Time $(\mathrm{n})$ & FIB $(\mathrm{g} / \mathrm{l})$ \\
\hline Blank group & 10 & $16.009 \pm 1.48$ & $20.06 \pm 1.76$ & $32.54 \pm 3.06$ & $1.87 \pm 0.15$ \\
Blood stasis group & 10 & $15.18 \pm 1.42$ & $18.78 \pm 1.69$ & $30.33 \pm 3.11$ & $1.93 \pm 0.16$ \\
Stroke group & 10 & $15.09 \pm 1.51$ & $18.79 \pm 1.72$ & $30.26 \pm 2.87$ & $1.97 \pm 0.16$ \\
Positive drug group & 10 & $15.62 \pm 1.39$ & $19.33 \pm 1.66$ & $31.33 \pm 2.44$ & $1.90 \pm 0.17$ \\
Shaomu group & 10 & $15.34 \pm 1.17$ & $18.94 \pm 1.48$ & $30.85 \pm 3.65$ & $1.95 \pm 0.13$ \\
Jiawei Shaomu & 10 & $15.47 \pm 1.84$ & $19.06 \pm 1.52$ & $31.29 \pm 3.74$ & $1.93 \pm 0.19$ \\
group & & & & & \\
\hline
\end{tabular}

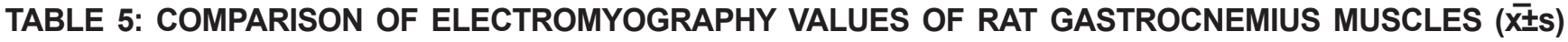
$(n=10)$

\begin{tabular}{lcc}
\hline Group & Sample size $(\mathrm{n})$ & Voltage values $(\mu \mathrm{V})$ \\
\hline Blank group & 10 & $85.56 \pm 7.62$ \\
Blood stasis group & 10 & $72.33 \pm 6.84^{\star}$ \\
Stroke group & 10 & $66.17 \pm 6.78^{\star \star}$ \\
Positive drug group & 10 & $80.44 \pm 6.71^{\star \star}$ \\
Shaomu group & 10 & $72.97 \pm 5.66^{\star}$ \\
Jiawei Shaomu group & 10 & $78.19 \pm 4.28^{\#}$ \\
\hline
\end{tabular}

Note: ${ }^{\wedge} p<0.01$ vs. blank group; * $p<0.01$ vs. blood stasis group; ${ }^{*} p<0.05$ vs. stroke group

TABLE 6: COMPARISON OF ANTIOXIDANT INDEXES IN RAT GASTROCNEMIUS MUSCLE $(\overline{X \pm})(n=10)$

\begin{tabular}{|c|c|c|c|}
\hline Group & Sample size $(n)$ & SOD (U/g) & MDA ( $\mu \mathrm{mol} / \mathrm{g})$ \\
\hline Blank group & 10 & $285.72 \pm 25.13$ & $65.15 \pm 7.18$ \\
\hline Blood stasis group & 10 & $152.57 \pm 18.56^{\triangle}$ & $80.33 \pm 7.23^{\Delta}$ \\
\hline Stroke group & 10 & $109.58 \pm 17.37^{\Delta \star}$ & $86.17 \pm 5.17^{\triangleleft}$ \\
\hline Positive drug group & 10 & $189.66 \pm 15.67^{\triangle \#}$ & $78.17 \pm 4.67^{\#}$ \\
\hline Shaomu group & 10 & $218.72 \pm 21.83^{\triangle \star \# \circ}$ & $72.14 \pm 5.78^{\text {太\# }}$ \\
\hline Jiawei Shaomu group & 10 & $239.64 \pm 20.64^{\Delta \star \# \circ}$ & $69.29 \pm 4.88^{\text {^\#० }}$ \\
\hline
\end{tabular}

Note: ${ }^{\wedge} \mathrm{p}<0.01$ vs. blank group, ${ }^{*} \mathrm{p}<0.01$ vs. blood stasis group, ${ }^{\sharp} \mathrm{p}<0.05$ vs. stroke group, ${ }^{\circ} \mathrm{p}<0.05$ vs. stroke group 
TABLE 7: COMPARISON OF INFLAMMATORY FACTORS IN RAT GASTROCNEMIUS MUSCLE $(\overline{\mathrm{x} \pm s)}(\mathrm{n}=10)$

\begin{tabular}{|c|c|c|c|}
\hline Group & Sample size $(\mathrm{n})$ & IL-1B (Pg/g) & TNF-a (ng/g) \\
\hline Blank group & 10 & $97.22 \pm 9.15$ & $58.65 \pm 5.21$ \\
\hline Blood stasis group & 10 & $122.17 \pm 10.65^{\Delta}$ & $82.33 \pm 7.18^{\Delta}$ \\
\hline Stroke group & 10 & $146.28 \pm 16.36^{\Delta \star}$ & $106.17 \pm 9.92^{\Delta *}$ \\
\hline Positive drug group & 10 & $108.41 \pm 10.26^{\star \#}$ & $63.54 \pm 6.83^{\star \#}$ \\
\hline Shaomu group & 10 & $112.85 \pm 13.26^{* \#}$ & $80.64 \pm 7.21^{\triangle \# \circ}$ \\
\hline Jiawei Shaomu group & 10 & $109.74 \pm 10.86^{\star \#}$ & $71.18 \pm 8.33^{\Delta \# \circ}$ \\
\hline
\end{tabular}

Note: ${ }^{\wedge} \mathrm{p}<0.01$ vs. blank group, ${ }^{*} \mathrm{p}<0.01$ vs. blood stasis group, ${ }^{\sharp} \mathrm{p}<0.05$ vs. stroke group, ${ }^{\circ} \mathrm{p}<0.05$ vs. stroke group

The results of transmission electron microscopy of gastrocnemius muscles of rats in the three groups are shown in fig. 1. As shown, the myofilaments of the blank group were clear and the mitochondria arranged normally; the myofilaments of the blood stasis group were deformed, appeared slight cavitation, the connection appeared interruption phenomenon and the mitochondria were enlarged; while in the stroke group, the clear myofilaments were extremely rare and the cavitation phenomenon was severe and autophagolysosomes appeared. While the muscle thread was significantly wider and mitochondria were reduced in the positive drug group, but better than in the model group. In the Shaomu group, myofilaments were broken, mitochondria were reduced and myofilaments were focally absent. In the Jiawei Shaomu group, mitochondria were not much degenerated, but myofilaments were disorganized.

The expression of genes related to the Nicotinic Acetylcholine Receptor (nAChR) signal pathway is shown in Table 8. As can be seen from Table 8, the expression of genes related to the $\mathrm{nAChR}$ signal pathway in the model group was significantly higher than that in the blank group ( $\mathrm{p}<0.01)$, the stroke group was higher than that in the blood stasis group $(\mathrm{p}<0.05)$, the drug treatment had an effect on down-regulating the expression of these genes $(p<0.05)$ and the downregulation effect of Traditional Chinese Medicine (TCM) was significantly better than that of the positive drug group $(p<0.05)$ and the effects of Jiawei Shaomu decoction was better than that of ordinary Shaomu decoction $(\mathrm{p}<0.05)$.

The expression of nAChR signal pathway related genes is shown in Table 9 and fig. 2. As can be seen, similar to the trend of gene expression, the contents of related proteins in the gastrocnemius muscle of rats in the model group were significantly higher than those in the blank group $(\mathrm{p}<0.05)$, the expression in the stroke group was higher than that in the blood stasis group $(p<0.05)$ and the drug treatment had the effect of downregulating the contents of related proteins $(\mathrm{p}<0.05)$ and the down-regulating effect of TCM was significantly better than that of the positive drug group $(p<0.05)$ and the effects of Jiawei Shaomu decoction was better than that of ordinary Shaomu decoction $(p<0.05)$.



Fig. 1: Effects on rat gastrocnemius muscle morphology 


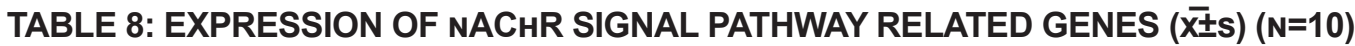

\begin{tabular}{|c|c|c|c|c|c|c|c|}
\hline Group & $\begin{array}{l}\text { Sample } \\
\text { size }(n)\end{array}$ & AChR & ERK & c-fos & Mek & Raf & Ras \\
\hline Blank group & 10 & $0.79 \pm 0.08$ & $1.59 \pm 0.11$ & $0.98 \pm 0.14$ & $0.73 \pm 0.09$ & $2.93 \pm 0.29$ & $0.68 \pm 0.03$ \\
\hline $\begin{array}{l}\text { Blood stasis } \\
\text { group }\end{array}$ & 10 & $4.70 \pm 0.24^{\Delta}$ & $6.66 \pm 0.52^{\Delta}$ & $6.25 \pm 0.38^{\Delta}$ & $6.30 \pm 0.56^{\Delta}$ & $7.79 \pm 0.57^{\Delta}$ & $7.37 \pm 0.51^{\triangleleft}$ \\
\hline Stroke group & 10 & $7.98 \pm 0.35^{\Delta \star}$ & $9.68 \pm 0.94^{\Delta \star}$ & $12.80 \pm 0.95^{\Delta \star}$ & $13.78 \pm 1.08^{\Delta \star}$ & $21.98 \pm 1.88^{\Delta \star}$ & $9.92 \pm 1.07^{\Delta \star}$ \\
\hline $\begin{array}{l}\text { Positive drug } \\
\text { group }\end{array}$ & 10 & $5.02 \pm 0.27^{\Delta \star \#}$ & $5.45 \pm 0.52^{\Delta \star \#}$ & $6.22 \pm 0.52^{\Delta \star \#}$ & $6.14 \pm 0.74^{\Delta \star \#}$ & $16.78 \pm 1.75^{\Delta \star \#}$ & $5.43 \pm 0.58^{\Delta \star \#}$ \\
\hline Shaomu group & 10 & $1.77 \pm 0.16^{\Delta \star \# \circ}$ & $1.93 \pm 0.15^{\Delta \star \# \circ}$ & $2.51 \pm 0.18^{\Delta \star \# \circ}$ & $2.87 \pm 0.28^{\Delta \star \# \circ}$ & $6.87 \pm 0.71^{\triangle \star \# \circ}$ & $2.54 \pm 0.38^{\triangle \star \# ० ~}$ \\
\hline $\begin{array}{l}\text { Jiawei } \\
\text { Shaomu group }\end{array}$ & 10 & $0.98 \pm 0.13^{\triangle \star \# \# \text { 约 }}$ & $1.79 \pm 0.17^{\triangle \star \# \circ}$ & $0.96 \pm 0.15^{\text {*\#ం하 }}$ & $1.48 \pm 0.13^{\Delta \star \# 0 \text { 约 }}$ & $2.86 \pm 0.19^{\text {*\#ం한 }}$ & $1.61 \pm 0.14^{\Delta \star \# 0 \text { 出 }}$ \\
\hline
\end{tabular}

Note: ${ }^{\wedge} p<0.01$ vs. blank group, * $\mathrm{p}<0.01$ vs. blood stasis group, ${ }^{\sharp} \mathrm{p}<0.05$ vs. stroke group, ${ }^{\circ} \mathrm{p}<0.05$ vs. stroke group, ${ }^{\star} \mathrm{p}<0.05$ vs. Shaomu group

TABLE 9: DETECTION OF PROTEIN CONTENTS RELATED TO nAChR SIGNAL PATHWAY $(\overline{\mathbf{x} \pm s)}(\mathrm{n}=10)$

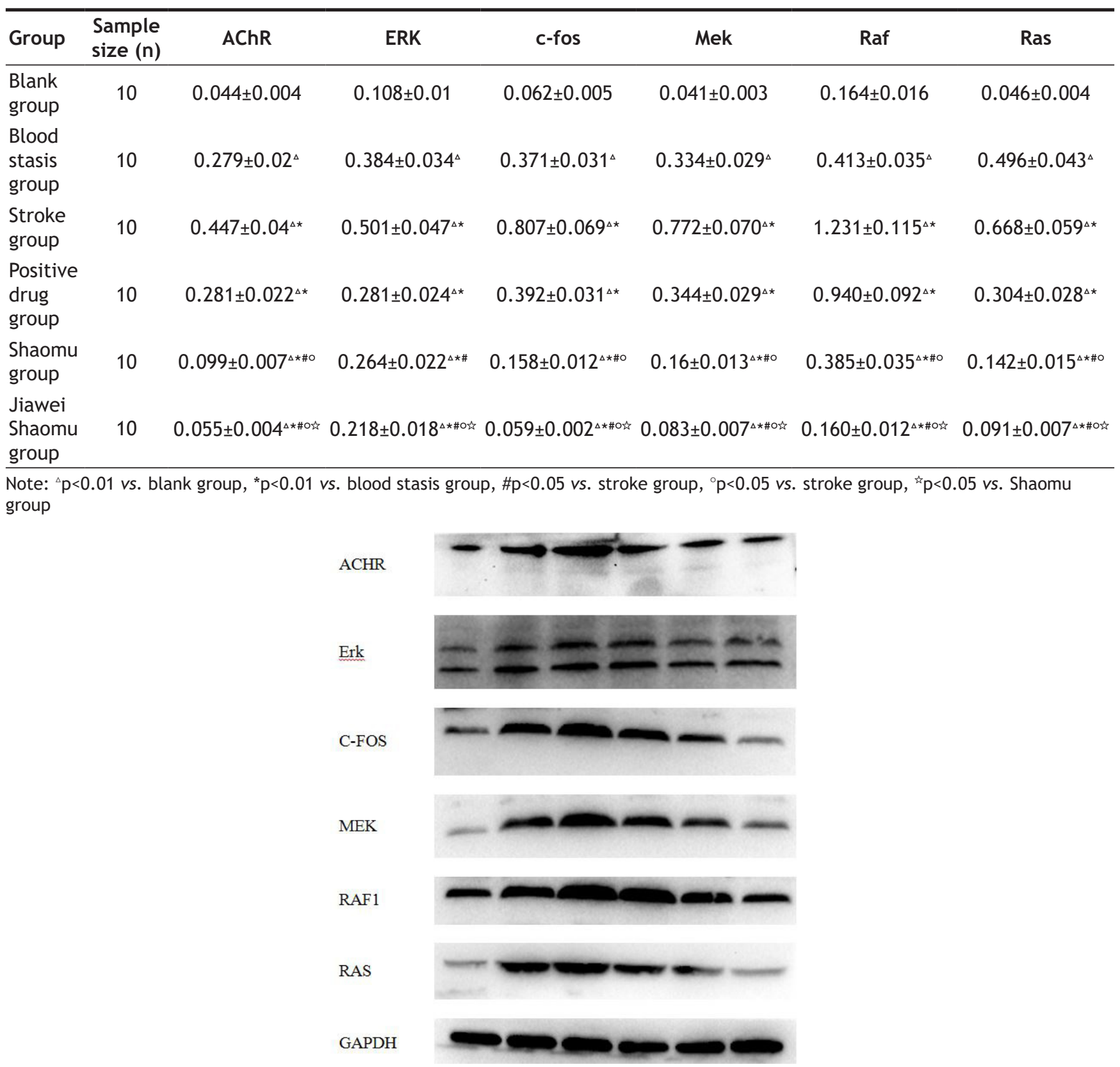

Fig. 2: Western blot of proteins related to AChR signal pathway in rat 
The central nervous system controls the body's skeletal muscles to accomplish physiological activities such as stretching and contraction by transmitting signals to the peripheral nervous system. After the onset of stroke, due to the inhibition of cerebral cortex and other higher nerve centers, the reflex threshold to dominant stretch appears to be reduced while the sensitivity of dominant motor neurons is enhanced, giving them a state of excitation, this manifests in patients as spasticity due to increased limb muscle tone. Traditional Chinese medicine considers "the blood of the internal knot as blood stasis", "the blood leaving the meridians as blood stasis", "chronic disease's entry into the collaterals as blood stasis" and "the hundred diseases are caused by grimy blood", that is, blood that is retained in Zang and $\mathrm{Fu}$ organs and tissues, or bleeding from visceral tissues, or blood that cannot be utilized by the body, or long-term diseases all fall under the category of blood stasis. Thrombosis and organ edema etc. in modern medicine also conform to the pathology of blood stasis syndrome, while western medicine considers that the occurrence of these diseases are all related to abnormal changes in blood flow, resulting in lesions in Zang and $\mathrm{Fu}$ organs and tissues. The blood that clogged in skeletal muscle is unable to supply nutrients to muscle cells and the peripheral nervous system that controls skeletal muscle, that is, 'lose nourishment' as traditional Chinese medicine refers to. Whereas the main role of muscles along the regular meridians is to interconnect the whole body as well as the limb muscles, so as to maintain the body's coordinated movement, when evil is full or positive Qi is deficient, the tendons are devitalized, producing "foot contracture urgency".

In the present experiment, increased expression of AChR protein was observed in both the blood stasis group and the stroke group, but perhaps the actual effect that this increase confers to patients is not exactly the same. For the blood stasis group, the activation of AChR signal pathway may help relieve the muscle wasting distress from muscular hypotrophy ${ }^{[7]}$, which may be the main reason why the inhibition of muscle tone and body weight was not significant in the rats of the blood stasis group. However, in contrast to the convalescent stroke, activation of the ERK signal pathway causes up-regulation of $\mathrm{AChR}^{[8]}$ at the sarcolemma and paradoxically, increases in muscle tone, leading to the appearance of post stroke muscle spasms. However, from the experimental results after administration treatment, each drug administration group can be inhibited to different degrees and the inhibition effect of the TCM group is better than that of the positive drug group, which indicates that Shaomu decoction and Jiawei Shaomu decoction can treat the muscle spasm signs caused by stroke by inhibiting the ERK signal pathway and down-regulating the concentration of AChR on the muscle cell membrane ${ }^{[9]}$.

Both Shaomu decoction and Jiawei Shaomu decoction have overall body conditioning effect and relieve muscle spasms from stroke sequelae. Shaomu decoction and Jiawei Shaomu decoction can effectively resist the inflammatory reaction that appears in gastrocnemius muscle after stroke by increasing antioxidant capacity. Shaomu decoction and Jiawei Shaomu decoction achieve their effects in treating muscle spasms after stroke by inhibiting the ERK signal pathway and downregulating AChR concentrations on the muscle cell membrane. Change is the essence of the prescription. Aiming at blood stasis type post stroke spasm, Jiawei Shaomu decoction shows better therapeutic effect than Shaomu decoction.

\section{Authors' contributions:}

Xiao Meng Wang and Quan E have contributed equally to this work.

\section{Conflict of interests:}

The authors declared no conflicts of interest.

\section{Acknowledgement:}

The worked was supported by Doctoral Research Fund Project of Hebei University of Traditional Chinese Medicine,(No.BSZ202014).

\section{REFERENCES}

1. Zainul Z, Heikkinen A, Koivisto H, Rautalahti I, Kallio M, Lin $\mathrm{S}$, et al. Collagen XIII is required for neuromuscular synapse regeneration and functional recovery after peripheral nerve injury. J Neurosci 2018;38(17):4243-58.

2. Min J, Qiaohong Q, Yulong C. Comparison of the vasomotor effects of total glucosides from Paeonia lactiflora Pallas and total glucosides from Radix Paeoniae rubra and study on its material basis. Pharmacol Clin Chin Materia Med 2018;34(5):71-6.

3. Jianjun Z, Yinfeng H, Lili W. Comparative study on Paeonia lactiflora Pallas, Paeoniae rubra and paeoniflorin and albiflorin std on the rehydration effect of a comprehensive exsanguination method on blood deficiency rats. Chin J Chin Materia Med 2013;38(19):3358-62.

4. Dorninger F, Herbst R, Kravic B, Camurdanoglu BZ, Macinkovic I, Zeitler G, et al. Reduced muscle strength in ether lipid-deficient mice is accompanied by altered development and function of the neuromuscular junction. J Neurochem 2017;143(5):569-83.

5. Xiong W, Feng X, Liu J, Chen W. Electroacupuncture for treatment of diabetic peripheral neuropathy: a systematic review of randomized controlled trials. J Tradit Chin Med Sci 2016;3(1):9-21. 
6. Leimbach F, Georgiev D, Litvak V, Antoniades C, Limousin P, Jahanshahi M, et al. Deep brain stimulation of the subthalamic nucleus does not affect the decrease of decision threshold during the choice process when there is no conflict, time pressure or reward. J CognNeurosci 2018;30(6):876-84.

7. Rogers RS, Nishimune H. The role of laminins in the organization and function of neuromuscular junctions. Matrix Biol 2017;57:86-105.

8. Liao F, Zheng Y, Cai J, Fan J, Wang J, Yang J, et al. Catestatin attenuates endoplasmic reticulum induced cell apoptosis by activation type 2 muscarinic acetylcholine receptor in cardiac ischemia/reperfusion. Sci Rep 2015;5(1):1-2.
9. Wang S, Seaberg B, Paez-Colasante X, Rimer M. Defective acetylcholine receptor subunit switch precedes atrophy of slow-twitch skeletal muscle fibers lacking erk 1/2 kinases in soleus muscle. Sci Rep 2016;7:45420.

This is an open access article distributed under the terms of the Creative Commons Attribution-NonCommercial-ShareAlike 3.0 License, which allows others to remix, tweak, and build upon the work non-commercially, as long as the author is credited and the new creations are licensed under the identical terms

This article was originally published in a special issue, "Diagnostic and Therapeutic Advances in Biomedical Research and Pharmaceutical Sciences"

Indian J Pharm Sci 2021:83(5) spl issue "288-298" 